\title{
Aprendizaje por Descubrimiento: Método Alternativo en la Enseñanza de la Física
}

\author{
Discovery Learning: Alternative Method in the Teaching of Physics \\ N. J. Castillo-Rodriguez , D. S. Giraldo-Santamaría ; A. Zapata-Gordon iD \\ DOI: https://doi.org/10.22517/23447214.24221 \\ Artículo de investigación científica y tecnológica.
}

\begin{abstract}
A dynamic circuit is carried out as a discovery learning method in the teaching of kinematics in 35 tenth grade students from the Hernando Caicedo School of La Paila - Valle Del Cauca, to strengthen the scientific thinking of the students. Classroom planning was designed based on discovery learning to verify the effectiveness of the kinematics teaching processes. A qualitative brain predominance test was applied to distribute the students according to the results in 5 groups that rotated through a circuit, performing 4 experiments and a feedback. Obtaining statistical values due to the interaction of several factors: brain predominance, compatible brain classroom planning and method of exploration and discovery, where initially in a diagnosis there was $45.42 \%$ of correct answers, while in the final evaluation there was $89.42 \%$ of correct answers, establishing significant learning and a normalized learning gain of 0.81 , thus supporting the effectiveness of the methodology used to teach kinematics in elementary school students. Through the data obtained, the effectiveness of classroom planning in support of the processes of teaching physics in elementary school students is revealed.
\end{abstract}

Index Terms - Brain Dominance, Learning, Discovery, Hake Factor, Teaching, Physics.

Resumen-Se realiza un circuito dinámico como método de aprendizaje por descubrimiento en la enseñanza de la cinemática en 35 estudiantes de grado décimo del Colegio Hernando Caicedo de La Paila - Valle Del Cauca, para fortalecer el pensamiento científico de los educandos. Se pasó a diseñar una planeación áulica con base en el aprendizaje por descubrimiento para verificar la eficacia de los procesos de enseñanza de la cinemática. Se aplicó una prueba cualitativa de predominancia cerebral para distribuir los estudiantes de acuerdo a los resultados en 5 grupos que rotaron por un circuito realizando 4 experimentos y una retroalimentación. Obteniendo valores estadísticos debido a la interacción de varios factores: predominancia cerebral, planeación áulica cerebro compatible y método de exploración y descubrimiento, donde inicialmente en un diagnóstico se tuvo el

This manuscript was sent on February 10, 2020 and accepted on November 23,2020 . Este trabajo es producto de una investigación realizada en una de las asignaturas de la Maestría en Enseñanza de la Física, de la Universidad Tecnológica de Pereira (UTP) y ejecutada en la asignatura de física en grado décimo del Colegio Hernando Caicedo.

N. J. Castillo-Rodriguez es Magíster en Instrumentación Física y Química Industrial egresada de la UTP. Actualmente es estudiante de la Maestría en Enseñanza de la Física en la UTP. También, es docente e investigadora de la UTP.(e-mail: nancycastillo@utp.edu.co).
45.42\% de aciertos, mientras que en la evaluación final se tuvo un $\mathbf{8 9 . 4 2 \%}$ de respuestas correctas, estableciendo un aprendizaje significativo y una ganancia de aprendizaje normalizada de $\mathbf{0 . 8 1}$, respaldando de esta manera la eficacia de la metodología empleada para la enseñanza de la cinemática en estudiantes de básica secundaria. Por medio de los datos obtenidos se pone en manifiesto la efectividad de la planeación áulica en apoyo a los procesos de la enseñanza de la física en estudiantes de básica secundaria.

Palabras claves - Aprendizaje, Descubrimiento, Factor Hake, Enseñanza, Física, Predominancia Cerebral.

Resumo - É realizado um circuito dinâmico como método de aprendizagem por descoberta no ensino de cinemática em 35 alunos do décimo ano da Escola Hernando Caicedo de La Paila Valle Del Cauca, para fortalecer o pensamento científico dos alunos. $O$ planejamento da sala de aula foi elaborado com base na aprendizagem por descoberta para verificar a eficácia dos processos de ensino da cinemática. Um teste qualitativo de predominância cerebral foi aplicado para distribuir os alunos de acordo com os resultados em 5 grupos que rodaram em um circuito, realizando 4 experimentos e um feedback. Obtenção de valores estatísticos devido à interação de vários fatores: predomínio cerebral, planejamento de sala de aula cerebral compatível e método de exploração e descoberta, onde inicialmente em um diagnóstico houve $45,42 \%$ de acertos, enquanto na avaliação final houve $89,42 \%$ de respostas corretas, estabelecendo uma aprendizagem significativa e um ganho de aprendizagem normalizado de 0,81 , apoiando assim a eficácia da metodologia utilizada para o ensino de cinemática em alunos do ensino fundamental. Por meio dos dados obtidos, revela-se a eficácia do planejamento de salas de aula no suporte aos processos de ensino de física em alunos do ensino fundamental.

D. S. Giraldo-Santamaría es Ingeniero Físico de la UTP. Actualmente es estudiante de la Maestría en Enseñanza de la Física en la UTP y docente del Colegio Hernando Caicedo en la asignatura de física.

(e-mail: dayangiraldo-1995@utp.edu.co).

A. Zapata-Gordon es Magister en Ingeniería Aeronáutica de la UTP. Actualmente es estudiante de la Maestría en Instrumentación Física de la UTP. También, es docente e investigadora de la UTP.

(e-mail: niko@utp.edu.co). 
Palavras Chave - Aprendizado, Descoberta, Fator de Pescada, Ensino, Física, Predominância do Cérebro.

\section{INTRODUCCIÓN}

$\mathrm{U}$ NO de los estudios de mayor alcance en el desarrollo educativo el día de hoy es el aprendizaje por descubrimiento, donde el contenido a ser aprendido, no se facilita en su forma final, sino que tiene que ser descubierto por el estudiante mismo, lo que requiere una actuación activa por parte del estudiante [1], que le permita aplicar lo experimentado en situaciones nuevas [2].

Existen diferentes maneras de descubrimiento, desde un descubrimiento autónomo, hasta un descubrimiento guiado y orientado por el docente; siendo este último el más utilizado en los métodos de enseñanza y aprendizaje [3].

Los métodos de la enseñanza por descubrimiento guiada, implica proporcionar al estudiante de oportunidades para utilizar dinámicamente los objetos y transformarlos por la acción directa, así como actividades de estudio que permitan al estudiante buscar, explorar, identificar y calcular una variable desconocida. Estas oportunidades, no solo incrementan el conocimiento de los estudiantes acerca del tema, sino que estimulan su curiosidad y los ayudan a desarrollar estrategias para aprender a partir del descubrir los conceptos inherentes en el conocimiento [3].

El aprendizaje por descubrimiento, defendido por Bruner, parte del constructivismo, el cual plantea que el modo de aprender depende de la construcción de competencias por parte de los estudiantes siendo a la vez esta relación una forma activa de adquirir el conocimiento [4].

Frisancho S, sustenta que, "El aprendizaje por descubrimiento según Bruner son técnicas de enseñanza por el método del descubrimiento. Para Bruner, el aprendizaje por descubrimiento es a la vez un objetivo de la educación y una práctica de su teoría de la instrucción" [5].

Shulman citado por Hormaza M. y Roca J, manifiesta que, "el aprendizaje por descubrimiento es un procedimiento antiguo reconocido por su gran efectividad en la sociedad. Permite al estudiante hacer generalizaciones entre casos concretos de una misma clase y por poder discriminar entre los casos que son y que no son de esa clase [5].

Rivero (2004) señala que los experimentos de demostración son necesarios para que los estudiantes adviertan que la Física es una Ciencia Natural, y que cada teoría debe, finalmente, basarse en las repuestas que la naturaleza proporciona a las preguntas, formuladas de manera adecuada por medio de los experimentos [6].

Walter L. Arias G. y Adriana Oblitas H. (2014) Realizaron un estudio sobre "Aprendizaje por descubrimiento vs. Aprendizaje significativo: Un experimento en el curso de historia de la psicología" donde compararon el rendimiento académico de dos grupos de estudiantes universitarios del Programa Profesional de Psicología de la Universidad Católica San Pablo, considerando que un grupo fue instruido con el modelo de aprendizaje por descubrimiento de Bruner en el curso de historia de la psicología I, y otro grupo fue instruido con el modelo de aprendizaje significativo por recepción de Ausubel [7].

\section{MATERIALES Y MÉTODOS}

\section{A. Materiales}

Cronómetro, fichas de dominó, cinta métrica, cinta aislante, arandelas, tornillos, nylon, pelota de plástico.

\section{B. Equipos Utilizados}

Computador, Laboratorios virtuales (internet)

\section{Método}

El estudio se llevó a cabo a partir de una población de 35 estudiantes de grado décimo del Colegio Hernando Caicedo de La Paila - Valle Del Cauca, en la asignatura de física.

La metodología es una propuesta realizada por los autores de la investigación para la enseñanza de la cinemática, basados en la planificación áulica propuestos por Merker y Waipan que se encuentra en el capítulo 8 de su escrito "el cerebro adolescente va al aula" [8], en donde cada una de las diferentes instancias quedan plasmadas a continuación:

\section{Caracterización de predominancia cerebral:}

Se utiliza un test diseñado y validado para identificar en el estudiante su predominancia cerebral denominado Revelador del Cociente Mental Tríadico (RCMT), donde se utiliza un cuestionario online para realizar la segmentación y etiquetar al mismo tiempo al estudiante líder, el estudiante analítico y el estudiante creativo.

\section{Diagnóstico de conocimientos previos:}

Se aplica una evaluación inicial (pre-test), mediante un formulario online diseñado previamente con 20 preguntas de selección múltiple sobre conceptos básicos de la cinemática como el movimiento rectilíneo uniforme (MRU), movimiento rectilíneo uniformemente acelerado (MRUA), caída libre y movimiento parabólico. El docente no resuelve inquietudes en este apartado de la clase.

\section{Desarrollo del contenido temático y actividades áulicas:}

Se tiene un grupo de estudiantes que previamente han trabajado los conceptos de la cinemática con un método de enseñanza tradicional que favorece aprendizajes repetitivos y memorísticos. Poco comprensivos del tema abordado, se propone a los estudiantes participar en un modelo de enseñanza constructivista para profundizar en la comprensión de los temas a abordar.

-Apertura: Se realiza un seteo emocional proyectando un video denominado "tolerancia en el puente" (YouTube) con el 
objetivo de captar el interés, la curiosidad y disposición para el aprendizaje por parte de los estudiantes [8], además es un ejemplo de cómo trabajar en equipo para realizar posteriormente las prácticas de los experimentos.

Con el apoyo de una presentación en la plataforma de Genially se realiza un acercamiento a los conceptos que integran la cinemática contempla las siguientes preguntas: ¿Qué es el movimiento rectilíneo uniforme?, ¿Qué es la velocidad?, ¿Qué es la aceleración?, ¿Por qué los objetos caen hacia abajo?, ¿Qué es un movimiento parabólico?, donde posteriormente se indica a los estudiantes que respondan de una forma escrita en una hoja en blanco dichas preguntas, seguidamente se presentan en diapositivas, las respuestas a aquellas preguntas por parte del docente, implicando al mismo tiempo a generar una comparación entre las respuestas y crear un desequilibrio cognitivo que conlleva al estudiante a percibir la importancia de aprender y apropiar los conceptos de la cinemática, activando de esta forma la disposición para el aprendizaje [9].

-Conformación de equipos de trabajo: Apoyados con los resultados obtenidos en el RMCT, pues según W. De Gregori [10], se construyeron los grupos de tal forma que se conserve el equilibrio entre ellos, y exista al menos un integrante por cada tipo de predominancia cerebral. Por ende, se propone 5 equipos de trabajo conformados por, 1 líder (Predominancia Central), 2 analíticos (Predominancia Izquierda) y 4 creativos (Predominancia Derecha).

-Actividades experimentales: el enfoque de la clase se desarrolló en torno a la enseñanza de la cinemática, implementando experimentos de bajo costo, donde los estudiantes puedan interactuar directamente con el conocimiento a adquirir; donde se pueda llegar a presentar una coherencia con el fenómeno de estudio, y el material potencialmente significativo [9]. Se realizan 5 guías prácticas que orientan al estudiante durante el proceso de la misma a como desenvolverse y adquirir el conocimiento pertinente de los temas propuestos. Se dispone de un sitio amplio (aula máxima) donde se ubican 5 puestos demarcados en orden de numeración del 1 al 5 para realizar las prácticas como se observa en la figura 1.

En esta sesión los docentes orientan y guían el proceso experimental de cada grupo, con el fin de promover un aprendizaje significativo donde exista interacción con los demás [9], y fomentando que entre los integrantes de cada grupo comprueben, discutan o rechacen hipótesis propias planteadas de acuerdo a los fenómenos que se observan en la práctica, por último se les recomienda que tomen nota de los datos en los experimentos para realizar un análisis de las posibles causas de dichos fenómenos físicos asociados al movimiento cinemático.

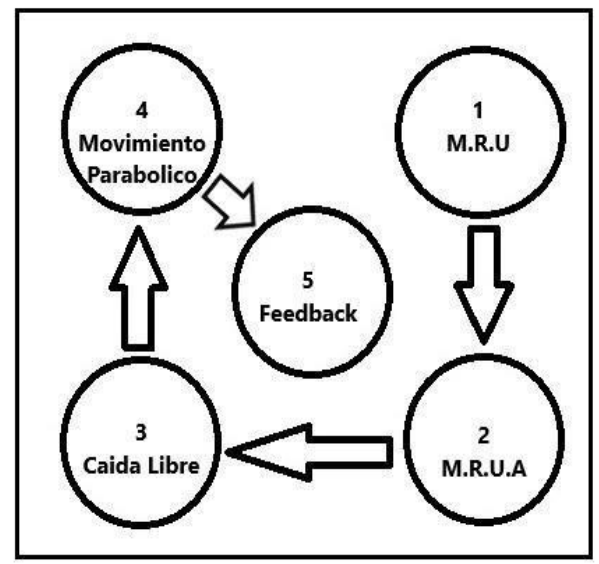

Fig. 1. Estrategia de trabajo, cada número representa un experimento y los grupos de trabajo rotan de manera ordenada del 1 al 5.

-Estrategia para la construcción social del conocimiento: debido a que los conceptos de cinemática necesitan de unas definiciones físicas, matemáticos y un aprendizaje paulatino de los temas, se propone que cada grupo recorra de una manera ascendente los diferentes experimentos tipo circuito, empezando en el sitio 1 y terminando en el sitio 5. Cada experimento tiene su guía práctica, con el objetivo que se demuestre de forma lúdica cada concepto de los diferentes tipos de movimiento de la cinemática. El experimento 1 comienza tratando el movimiento más elemental (MRU) mediante el cálculo e interpretación de la velocidad de una reacción en cadena consistente en una hilera de fichas de dominó en caída sucesiva, en función de la separación existente entre ellas. Luego se realiza el experimento 2 con un movimiento acelerado (MRUA) sujetando dos masas con nylon, una masa mucho mayor que la otra y se deja caer libremente, mientras la masa pequeña se mueve con una aceleración constate sobre una superficie. Siguiendo con el tercer experimento relacionado con la caída libre, interpretando la relación entre la distancia recorrida con respecto al tiempo por una pelota que se deja caer a una altura dada, verificando que la aceleración del movimiento es la gravedad, continuando con el experimento 4 movimiento parabólico donde el estudiante utiliza una simulación en la página PHET para determinar el angulo de lanzamiento y velocidad inicial para impactar la bala de un cañón en el blanco. Sin embargo, los experimentos están diseñados para que se logren desarrollar simultáneamente en una duración de 20 minutos, y el estudiante pueda entender el concepto trabajado en cada experimento.

-Socialización de los experimentos y conceptos: Los conceptos básicos de cinemática abordados normalmente por el plan de estudio de secundaria se aprende mediante una clase magistral; con esta propuesta de aprendizaje por descubrimiento se motiva a que los estudiantes asuman el reto y realicen un breve análisis de lo que se evidencia en cada experimento implementado. Para este caso, entre los miembros del equipo de trabajo plantean preguntas sobre el fenómeno físico observado, y mediante un análisis de datos y de gráficas, formulan las respuestas para después abrir un debate donde todos los estudiantes participen y defiendan la comprobación o 
refutación de dichas hipótesis. Esta socialización es de vital importancia para el proceso de aprendizaje, ya que al poner en evidencia lo que cada integrante experimentó, los estudiantes van asociando lo que realizó y observó con los conceptos de cinemática, lo que permite un aprendizaje significativo. Además, queda en plena evidencia el aprendizaje según la teoría socio-cultural, ya que cada rol dentro del grupo es importante y hace necesario la interacción con los demás para alcanzar el conocimiento nuevo [9] [11].

-Refuerzo teórico: Después de realizar los 4 primeros experimentos, se propone realizar un refuerzo teórico en el sitio número 5, donde el orientador utiliza un lenguaje técnico, acorde al tema, y donde se relacione lo verificado en los experimentos. En este caso específico, en cinemática se realiza un análisis al comportamiento de los datos y gráficos. Además, En esta sesión se deben reforzar los siguientes conceptos: desplazamiento, velocidad, aceleración, movimiento en un eje, movimiento en dos ejes, movimiento con velocidad constante y movimiento con aceleración constante.

-Evaluación: después de culminado el desarrollo de la sesión del circuito completo por los 5 equipos de trabajo, se aplica una evaluación final (pos-test). Este test es el mismo que se aplica antes de ejecutar la propuesta metodológica y se evalúa mediante la aplicación ZipGrade para optimizar los tiempos de análisis. El pre-test y el pos-test pueden tener la misma estructura, ya que se utilizarán para el análisis de la ganancia de aprendizaje por estudiante. Al comparar el pre-test con el postest se puede estimar qué conceptos pudo aprender y apropiar el estudiante gracias al proceso metodológico implementado en el aula [11].

-Actividad de cierre: Con el objetivo de que cada estudiante pueda identificar que sabía antes de la clase y que aprendió con esta metodología propuesta, es necesario realizar una actividad de cierre que permita culminar el proceso. Para este caso puntual, se le pidió al estudiante que haga una síntesis mediante palabras escritas, de los nuevos términos o conceptos aprendidos en la actividad.

-Actividad de retroalimentación: Para finalizar, se realiza una actividad de realimentación que permite al docente conocer las opiniones de sus estudiantes con respecto a la metodología implementada en la clase. Se dio instrucción a los estudiantes a escribir las opiniones y comentarios de la clase para enriquecer el proceso y dar cuenta al orientador como se sintieron sus aprendices.

\section{RESULTADOS Y DiscUSIÓN}

Esta metodología se diseñó y se pensó para aplicarla a estudiantes de educación media, o iniciando los primeros semestres de educación superior donde se imparten cursos de física. En este caso, la propuesta metodológica fue aplicada a un grupo de 35 estudiantes de grado décimo, en el colegio de La Paila - Valle Del Cauca. A continuación, se exponen los resultados obtenidos, producto de la presente investigación.
A-Caracterización del grupo y predominancia cerebral: La edad promedio de los estudiantes oscila entre los 15 años. Como se observa en la tabla 1 , se presenta un mayor número de estudiantes con predominancia cerebral derecha, mientras que un grupo minoritario son centrales e izquierdos.

Realizando una comparación en porcentajes se clasificó las predominancias como: Derecho (57\%), Izquierdo (29\%) y central (14\%). En la figura 2 se representa dicha distribución. Cada predominancia tiene un rol importante en ejecutar los experimentos, los estudiantes con una predominancia central tuvieron el rol del líder coordinando a su grupo de trabajo, los estudiantes con predominancia derecha tuvieron el rol de creativos realizando el montaje de los experimentos y finalmente los estudiantes con predominancia izquierda tuvieron un rol de analíticos realizando cálculos e interpretación de gráficas.

A raíz de estos resultados, se divide el grupo de estudiantes en 5 equipos de trabajos, conformados por un líder (centrales), 2 analísticos (izquierdos) y 4 creativos (derechos), cada integrante tiene su propio rol dentro del equipo. Estos resultados se respaldan por la carrera profesional que manifiestan los estudiantes querer estudiar (tabla 1). Además, es un reto para los docentes ya que de entrada no se tiene una disposición o afinidad por parte de la mayoría del grupo, quienes prefieren actividades relacionados con las bellas artes y humanidades.

\section{PREDOMINANCIA CEREBRAL}

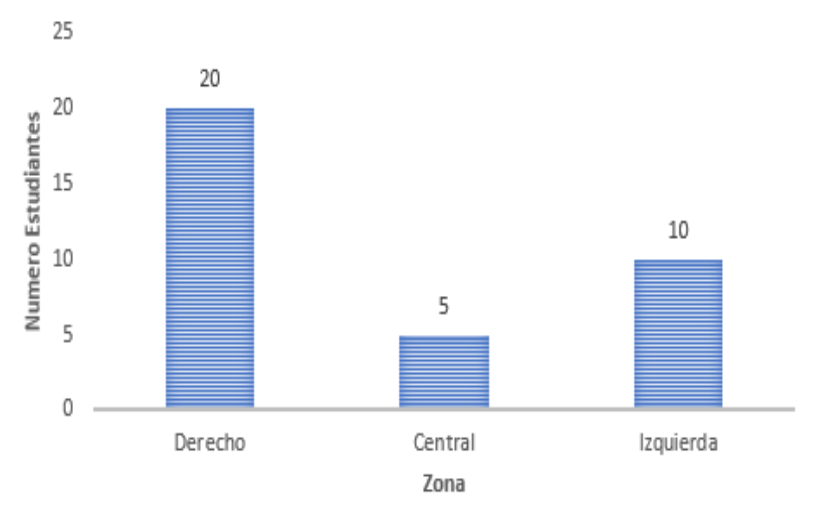

Fig. 2. Distribución de la predominancia cerebral del grupo.

TABLA I

RESULTADO REVELADOR COCIENTE MENTAL TRÍADICO PREDOMINANCIA CEREBRAL DE CADA ALUMNO

\begin{tabular}{ccc}
\hline \hline Estudiante & Predominancia & Profesión que le gustaría ejercer \\
\hline 1 & Derecho & Veterinaria \\
2 & Derecho & Abogacía \\
3 & Derecho & Psicología \\
4 & Derecho & Medicina \\
5 & Izquierdo & Ingeniería de petróleos \\
6 & Central & Enfermería \\
7 & Derecho & Música \\
8 & Izquierdo & Ingeniería Sistemas
\end{tabular}




\begin{tabular}{ccc}
9 & Derecho & Licenciatura Comunicación \\
10 & Derecho & Artes Plásticas \\
11 & Derecho & Abogacía \\
12 & Derecho & Licenciatura Humanidades \\
13 & Izquierdo & Matemáticas \\
14 & Central & Administración empresas \\
15 & Central & Administración empresas \\
16 & Derecho & Medicina \\
17 & Derecho & Medicina \\
18 & Derecho & Piloto \\
19 & Derecho & Veterinaria \\
20 & Derecho & Filosofía \\
21 & Central & Economía \\
22 & Derecho & Enfermería \\
23 & Derecho & Filosofía \\
24 & Izquierdo & Ingeniería de sistemas \\
25 & Derecho & Fisioterapia \\
26 & Izquierdo & Ingeniería Industrial \\
27 & Izquierdo & Contabilidad \\
28 & Izquierdo & Contabilidad \\
29 & Izquierdo & Ingeniería Mecánica \\
30 & Central & Administración empresas \\
31 & Derecho & Auxiliar administrativo \\
32 & Derecho & Diseño gráfico \\
33 & Izquierdo & Ingeniería Industrial \\
34 & Izquierdo & Economía \\
35 & Derecho & Humanidades \\
\hline
\end{tabular}

B. Análisis de aciertos por estudiante: Este estudio se muestra en la figura 3 , donde se aprecia la información que puede ser útil para identificar las falencias o dificultades de los estudiantes para responder las preguntas. Se puede hacer un contraste diferenciador sobre los conceptos que los educandos ya sabían, con los nuevos aprendidos durante la sesión de clase. A nivel general se pueden analizar estudiantes que de 20 preguntas, acertaron 4 en el pre-test, mientras que en el pos-test el mismo estudiante respondió acertadamente las 20 preguntas.

Dicha gráfica es un recurso ágil para contrastar los conocimientos previos de los conocimientos adquiridos después de ejecutar la propuesta metodológica.

C. Análisis del pre-test y pos-test tomando como referencia un sistema de evaluación tradicional: Si el número de preguntas acertadas en el pre-test y el pos-test se analizan tomando como referencia un sistema tradicional de evaluación, con una escala de calificación de 0 a 5, se obtienen los datos mostrados en la tabla 2. Teniendo en cuenta que según dicha escala de calificación, un examen se aprueba con una nota superior a 3,0 se concluye que sólo 4 estudiantes aprobaron el examen en el pre-test. Sin embargo, todos aprobaron la evaluación en el postest. Se determina que el promedio del grupo en el pre-test fue de 2,3. Mientras que para el pos-test, el promedio del grupo ascendió a 4,5. El promedio de notas del grupo mejora un $44 \%$ después de ejecutar la propuesta metodológica de aprendizaje por descubrimiento.

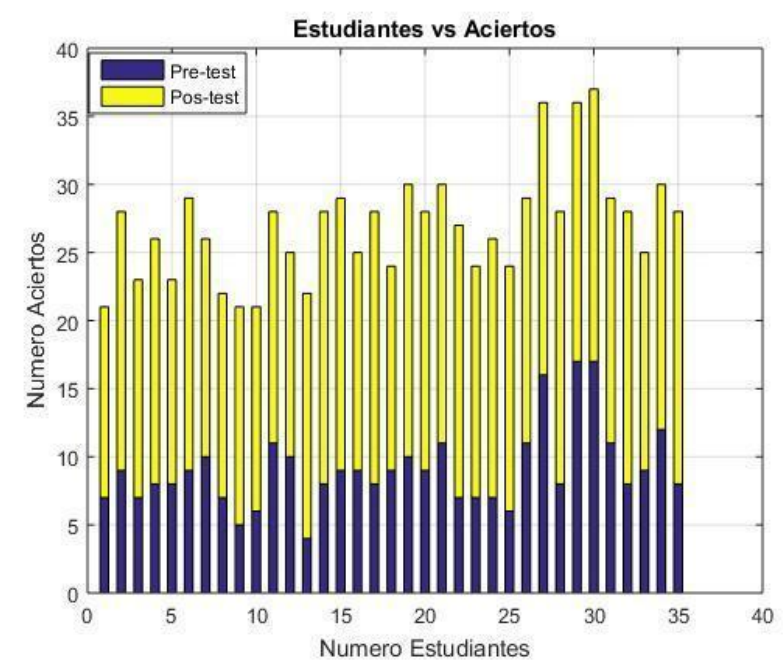

Fig. 3. Diagrama de barras que muestra el contraste entre el número de acierto por pregunta que se obtuvieron en el pre-test y en el pos-test por estudiante.

D. Análisis y cálculo del factor de Hake: Para realizar el análisis de la ganancia promedio normalizada o factor de Hake, se debe partir de la tabla 2 donde se discrimina el número de aciertos que obtuvo cada estudiante en el pre-test y en el postest [11]. Se debe tener en cuenta que el número máximo de aciertos posibles es 20 , ya que el test consta de 20 preguntas. Según la tabla 2, el número total de aciertos obtenidos en el pretest fue de 318 , y en el pos-test fue de 626 , de 700 posibles.

Para determinar la ganancia promedio normalizada $\langle\mathrm{G}\rangle$ se llevó a cabo el siguiente procedimiento:

Primero se calcula la puntuación máxima que se puede alcanzar a nivel de grupo, el puntaje obtenido en el pre-test, $\mathrm{y}$ el puntaje obtenido en el pos-test [11] [12]. Donde $N$ es el número de estudiantes (35), $P$ es el número de preguntas del test (20), TPRE es número total de aciertos que se obtuvieron en el pre-test a nivel grupal (318), y TPOS es número total de aciertos que se obtuvieron en el pos-test a nivel grupal (626).

$$
\begin{gathered}
P_{\max }=N X P=35 \times 20=700 \\
P_{P R E}=T_{P R E} X 100=318 \times 100=31800 \\
P_{P O S}=T_{P O S} X 100=626 \times 100=62600
\end{gathered}
$$

Después se obtuvieron los puntajes de respuestas correctas para el pre-test y el pos-test en porcentajes [11].

$$
\begin{aligned}
& <\operatorname{Pretest}(\%)>=\frac{P_{P R E}}{P_{\max }}=\frac{31800}{700}=45,42 \% \\
& <\operatorname{Postest}(\%)>=\frac{P_{P O S}}{P_{\max }}=\frac{62600}{700}=89,42 \%
\end{aligned}
$$

Para obtener la ganancia de aprendizaje normalizada $\langle\mathrm{G}\rangle$ se reemplazan los valores obtenidos anteriormente en la siguiente ecuación:

$$
<G>=\frac{<\text { Postest }(\%)>-<\operatorname{Pretest}(\%)>}{1-<\operatorname{Pretest}(\%)>}
$$




$$
<G>=\frac{0,8942-0,4542}{1-0,4542}=0,81
$$

Hake propone tres categorías para la ganancia promedio normalizada, que indican el grado de efectividad de la estrategia de enseñanza implementada en el aula de clase [11]:

- Ganancia alta: 0,7 < $(<G>) \leq 1$

- Ganancia media: 0,3<(<G>) $\leq 0,7$

- Ganancia baja: $(<\mathrm{G}>) \leq 0,3$

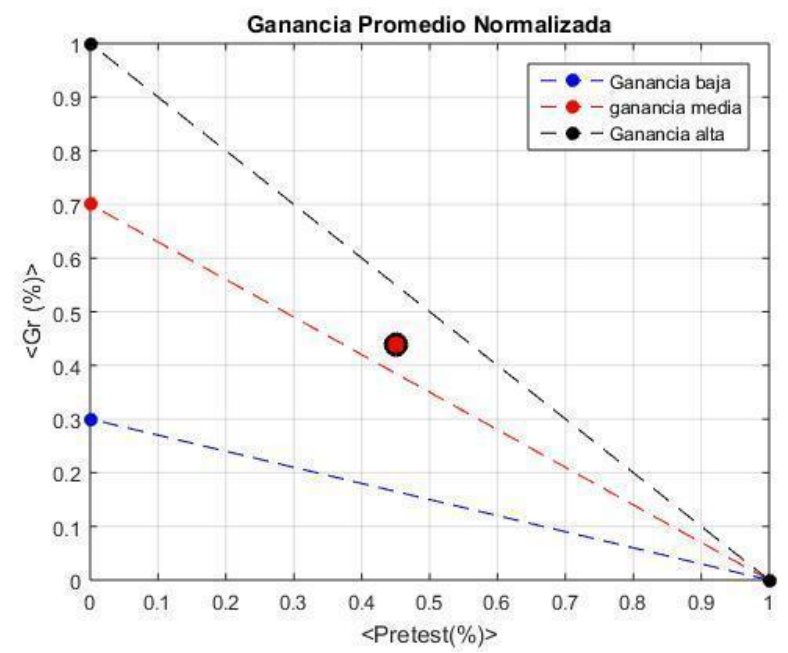

Fig. 4. Ganancia real promedio contra promedio del pre-test. Se muestran también las tres regiones de interés según Hake. El punto rojo representa la ganancia promedio que obtuvo el grupo, es decir, el contraste entre los conocimientos previos de los estudiantes con la ganancia de aprendizaje que se obtuvo después de aplicar la actividad áulica.

En síntesis, el test de Hake relaciona el conocimiento previo del estudiante cuando llega a clase, con el conocimiento que aprendió durante la clase y con lo que debía aprender [13]. El resultado de la ganancia promedio normalizada $\langle\mathrm{G}\rangle=0,81$ indica que el factor de ganancia de aprendizaje se encuentra en la zona de ganancia alta, según las regiones de ganancia propuestas por Hake [12]. Esto revela que la metodología utilizada en la clase de aprendizaje por descubrimiento tuvo una comprensión conceptual alta, es decir, que el proceso de enseñanza-aprendizaje fue significativo para los estudiantes y que el conocimiento se apropió positivamente.

Para comprender mejor este resultado, se muestra en la figura 4 la gráfica de zonas de ganancia propuesta por Hake, donde se ubica en la zona o nivel de alta ganancia, la ganancia promedio normalizada obtenida en esta investigación. El eje horizontal representa el puntaje promedio del pre-test (normalizado) y el eje vertical representa la ganancia real promedio (normalizada) [11] [12].
TABLA II

Número De Aciertos Y CALIFICACIONES ObTENIDA En El PRE-TEST Y Pos-Test POR CADA ESTUDIANTE

\begin{tabular}{|c|c|c|c|c|}
\hline \multirow[t]{2}{*}{ Estudiante } & \multicolumn{2}{|c|}{ Pre-Test } & \multicolumn{2}{|c|}{ Pos-Test } \\
\hline & Aciertos & Calificación & Acierto & Calificación \\
\hline 1 & 7 & 1,8 & 14 & 3,5 \\
\hline 2 & 9 & 2,3 & 19 & 4,8 \\
\hline 3 & 7 & 1,8 & 16 & 4,0 \\
\hline 4 & 8 & 2,0 & 18 & 4,5 \\
\hline 5 & 8 & 2,0 & 15 & 3,8 \\
\hline 6 & 9 & 2,3 & 20 & 5,0 \\
\hline 7 & 10 & 2,5 & 16 & 4,0 \\
\hline 8 & 7 & 1,8 & 15 & 3,8 \\
\hline 9 & 5 & 1,3 & 16 & 4,0 \\
\hline 10 & 6 & 1,5 & 15 & 3,8 \\
\hline 11 & 11 & 2,8 & 17 & 4,3 \\
\hline 12 & 10 & 2,5 & 15 & 3,8 \\
\hline 13 & 4 & 1,0 & 18 & 4,5 \\
\hline 14 & 8 & 2,0 & 20 & 5,0 \\
\hline 15 & 9 & 2,3 & 20 & 5,0 \\
\hline 16 & 9 & 2,3 & 16 & 4,0 \\
\hline 17 & 8 & 2,0 & 20 & 5,0 \\
\hline 18 & 9 & 2,3 & 15 & 3,8 \\
\hline 19 & 10 & 2,5 & 20 & 5,0 \\
\hline 20 & 9 & 2,3 & 19 & 4,8 \\
\hline 21 & 11 & 2,8 & 19 & 4,8 \\
\hline 22 & 7 & 1,8 & 20 & 5,0 \\
\hline 23 & 7 & 1,8 & 17 & 4,3 \\
\hline 24 & 7 & 1,8 & 19 & 4,8 \\
\hline 25 & 6 & 1,5 & 18 & 4,5 \\
\hline 26 & 11 & 2,8 & 18 & 4,5 \\
\hline 27 & 16 & 4,0 & 20 & 5,0 \\
\hline 28 & 8 & 2,0 & 20 & 5,0 \\
\hline 29 & 17 & 4,3 & 19 & 4,8 \\
\hline 30 & 17 & 4,3 & 20 & 5,0 \\
\hline 31 & 11 & 2,8 & 18 & 4,5 \\
\hline 32 & 8 & 2,0 & 20 & 5,0 \\
\hline 33 & 9 & 2,3 & 16 & 4,0 \\
\hline 34 & 12 & 3,0 & 18 & 4,5 \\
\hline
\end{tabular}

\section{CONCLUSIONES}

Los análisis encontrados demuestran que la estrategia de aprendizaje por descubrimiento más una propuesta de intervención áulica cerebro compatible aplicada a estudiante de educación secundaria o media vocacional no solo fortalece la apropiación del conocimiento, también conllevan al mejoramiento, la eficacia, la disposición por aprender y tener una afinidad por las ciencias físicas.

Esta investigación es una clara contribución de la eficiencia de la aplicabilidad del modelo de enseñanza-aprendizaje por descubrimiento en el área de la física para asimilar los conceptos de la cinemática en estudiantes de básica secundaria o media vocacional.

La ganancia promedio Normalizada fue de 0.81 demostrando que el método de aprendizaje es de gran importancia y colocando en manifiesto el rol de los estudiantes según su pre dominancias cerebrales y funciones dentro de un equipo de trabajo para la organización y contextualización de los conocimientos adquiridos a partir del aprendizaje por descubrimiento guiado. 


\section{REFERENCES}

[1] M. y. Zea, "Estrategias de enseñanza 1 basadas en un enfoque," Ciencias de la educación, vol. 2, pp. 69-90. ISSN: 0798-0329, 2004.

[2] J. S. Bruner, Toward a Theory of Instruction, Cambridge: Belkapp press.ISBN 0-674-89701-3, 1966, p. 176.

[3] C. Palomino y M. Eleizalde, "Aprendizaje por descubrimiento y su eficacia en la enseñanza de la Biotecnología," Revista de investigación, vol. 34, nº 71. ISSN: 0798-0329, pp. 271-290, 2010.

[4] A. Camargo y C. Hederich, "Dos teorías cognitivas, Dos formas de significar, Dos enfoques para la enseñanza de la física," Psicogente, vol. 13, n' ISSN: 0124-0137. EISSN 2027-212X, pp. 329-436, 2010.

[5] J. Riveros, El método de descubrimiento guiado en el aprendizaje de análisis combinatorio en los alumnos del quinto grado de educación secundaria de la institución educativa "Chinchaysuyo",, Perú: Huancayo, 2014..

[6] H. Rivero, Estrategias de Enseñanza/Aprendizaje: investigaciones desde el CRINCEF., México: pimenta.DOI: 10.31560/pimentacultural/2019.652., 2004..

[7] W. Arias y A. Oblitas, "Aprendizaje por descubrimiento vs Aprendizaje significativo: Un experimento en el curso de historia de la psicología," Bol. Acad. Paulista de Psicología,, vol. 34, nº 87. ISSN: 1415-711X, pp. 455-471, 2014.

[8] W. Liliana y M. Alejo, El cerebro adolescente va al aula. Neuroeducación, adolescencia y escuela secundaria,, Buenos Aires,: ISBN 9789876671859., 2017..

[9] E. Pinilla, M. saenz y L. Vera, Reflexiones sobre la Educación Universitaria, Bogota: ISBN: 958-701-327-1, 2003.

[10] C. Pérez y S. M. Rodríguez, "El cerebro tríadico y su relación con la curiosidad, el trabajo en equipo y la explicación de fenómenos para el desarrollo de actitud científica," Rastros Rostros, vol. 17, no 31. doi: http://dx.doi.org/10.16925/ra.v17i31.1106, pp. 93-103, 2015.

[11] R. Hake, "Introduction, Interactive-engagement vs traditional methods: A six-thousand-student survey of mechanics test data for introductory physics," American Journal Physics, vol. 38, nº doi: 10.1119/1.18809, pp. 64-66, 1998..

[12] J. S. a. G. Stewart, "Correcting the Normalized Gain for Guessing the Normalized Gaid for," Physics Teacher, vol. 48, $\mathrm{n}^{\circ}$ 3. doi: 10.1119/1.3317458, pp. 194-196, 2010.

[13] J. I. Pozo, Teorías cognitivas del aprendizaje, Madrid: Morata. ISBN: $9788471123350,1987$.

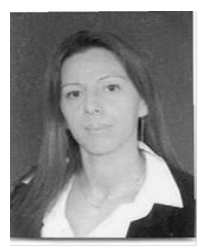

Nancy Janet Castillo Rodriguez, nació en Cali, Colombia, en 1978. Recibió los títulos de Tecnóloga Química, Química Industrial Y Magíster en Instrumentación Física por la universidad Tecnológica de Pereira (UTP), Pereira, en 1997,2008 y 2017. Actualmente Realiza sus estudios en Maestría en Enseñanza de la Física en la Universidad Tecnológica de Pereira, Desde 1998 se ha desempeñado como docente en el municipio de Pereira, Desde 2011 ha sido profesora en el Departamento de Física, UTP, y desde 2015 es profesora transitoria de medio tiempo. Sus intereses de investigación actuales incluyen proceso de tratamiento digital de imágenes en el campo de la medicina forense, identificación de fluidos biológicos en dictámenes de carácter forense y análisis de sustancias orgánicas. Pertenece al grupo de investigación Robótica Aplicada de la UTP.

ORCID: https://orcid.org/0000-0003-0856-0582

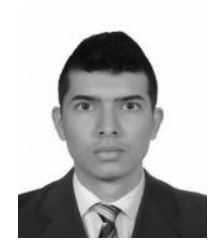

Dayan Steban Giraldo Santamaría, nació en Cartago, Colombia, en 1995. Recibió el título de Ingeniero Físico por la Universidad Tecnológica de Pereira (UTP), Pereira, en 2018. Actualmente realiza sus estudios en Maestría En Enseñanza De La Física en la Universidad Tecnológica de Pereira, Desde 2019 se ha desempeñado como docente en el área de ciencias naturales en el Colegio Hernando Caicedo, siendo actualmente profesor en la asignatura de física. Sus intereses de investigación actuales incluyen desarrollo de metodologías de aprendizaje significativo en la enseñanza de la física.

ORCID: https://orcid.org/0000-0002-3394-7191

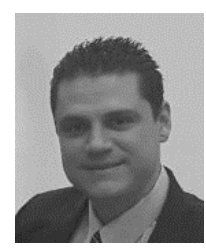

Adonaí Zapata Gordon, nació en Pereira, Colombia, en 1981, realizo sus estudios secundarios en formación Técnica en Mecánica Industrial en 1998, ese mismo año obtuvo el título de técnico en sistemas y diseñador gráfico. Ingeniero Mecánico en 2010, en la cual obtuvo la distinción como estudiante de excelencia de la Universidad Tecnológica de Pereira. Es Magister en Ingeniería Aeronáutica de la Universidad Autónoma de Nuevo León UANL (México), actualmente es estudiante de la Maestría en Instrumentación Física de la Universidad Tecnológica de Pereira UTP, Desde 1999 se ha desempeñado como docente del departamento de Risaralda. Desde el 2011 ha sido profesor/investigador de la Facultad de Tecnología en el Programa de Ingeniería Mecatrónica, desde el 2016 de la Facultad de Ingeniería Mecánica y desde el 2017 profesor de la Maestría en Enseñanza de la Física todos de la UTP. Sus intereses de investigación, innovación, extensión y desarrollo tecnológico actuales incluyen, ingeniería aeronáutica, robótica, materiales compuestos y experimentación en la física. Pertenece al Grupo de Investigación Robótica Aplicada y Grupo de Investigación Mecabot haciendo trabajo colaborativo en la UTP.

ORCID: https://orcid.org/0000-0001-8021-8576 